\title{
Genetic Divergence in Niger (Guizotia abyssinica (L.f) Cass)
}

\author{
Shubhangi G.Patil ${ }^{1}$, V. V. Bhavsar ${ }^{2 *}$, Shweta D. Deokar ${ }^{3}$ and V. S. Girase ${ }^{4}$ \\ Department of Agricultural Botany, College of Agriculture, \\ Dhule-424 004 (MPKV), (M.S.), India \\ *Corresponding author
}

\section{A B S T R A C T}

\section{Keywords}

Genetic diversity, D2 value, cluster, genotype

\section{Article Info}

Accepted:

20 August 2019

Available Online:

10 September 2019
An experiment was undertaken by utilizing forty five Niger genotypes for twelve yield and yield contributing characters to assess genetic divergence. The analysis of variance has shown that there was significant variation among the genotypes in all the traits. The multivariate analysis carried out using MahalanobisD ${ }^{2}$-statistics, indicated wider genetic diversity in the genotypes of niger. Out of ten cluster formed, cluster III was largest with twelve genotypes, followed by cluster I with eleven genotypes, cluster II and V with six genotypes, cluster VIII with five and IV, VI, VII, IX, X were mono-genotypic. The clustering pattern indicated absence of relationship between genetic diversity and geographical origin of the genotypes. The maximum inter cluster distance was observed between cluster II and X $\left(D^{2}=16.87\right)$ while, lowest divergence was noticed between cluster VII and $\mathrm{X}\left(\mathrm{D}^{2}=4.11\right)$. Maximum intra cluster distance observed within cluster $\mathrm{V}$ $\left(D^{2}=6.76\right)$ while lowest intra cluster distance was observed within cluster $I\left(D^{2}=5.16\right)$. The variance for cluster means were high for number of seeds per capitula (29.70), followed by number of secondary branches per plant (16.26), number of primary branches per plant (13.54), 1000 seed weight (12.53), number of capitula per plant (12.32) and was low for plant height, days to maturity, diameter of capitula, protein content, seed yield per plant, oil content, days to $50 \%$ flowering. Based on inter-cluster distances, cluster mean and per se performance, and divergence class the genotypes viz., DHLN-17, DHLN-18, DHLN-26, DHLN-29, DHLN-39, DHLN-41, DHLN-42, DHLN-44 were distinct and diverse and can be classified as promising genotypes. These seven genotypes can be used for inter-crossing to obtain heterosis and also wider variability in Niger. Hybridization between the genotypes of cluster II with the genotypes of cluster X may result in exploiting more heterosis with maximum genetic divergence and are likely to produce desirable transgressive segregants in segregating generations for further crop improvement.

\section{Introduction}

Niger (Guizotia abyssinica (Lf) Cass) is named after the French historian Guizot. It belongs to the family Compositae/Asteraceae, tribe Helianthoides and subtribe Verbeninae. It is an oilseed crop cultivated in Indian subcontinents and East African Countries. It is self incompatible crop having diploid chromosome $2 n=30$. It is minor crop grown mostly in India and Ethiopia where it is known as Ram til, Kala til, Karala, Gurellu, Tilangi and Neuk, Noog and Nug. Niger is the native of highlands of Ethiopia and originated from 
G. scabre subsp. Schimperi, where it is a common weed in fields with grown Niger. The wild form has oil content of 24 to $35 \%$, while the cultivated Niger has 36 to $42 \%$ oil with fatty acid composition of 75 to $80 \%$ linoleic acid, 7 to $8 \%$ palmitic and stearic acid and 5 to $8 \%$ oleic acid. Indian Niger oil reported higher in oleic acid (25\%) and lower in linoleic acid (55\%). Niger has $10-30 \%$ protein content. Niger is a dicotyledonous herb, moderately to well branched, grows up to two meter tall. Niger plant like other compositae is highly cross pollinated oilseed crop mostly grown on marginal and sub marginal land. In India the Niger is grown on an area of 2.61 lakh ha mainly during Kharif, and average productivity in India is $321 \mathrm{~kg} / \mathrm{ha}$ with production 0.84 lakh tonnes. India is the largest exporter of Niger in the world to USA, Netherland, Italy, Germany, Belgium, and Spain is the regular buyer. Whereas, USA is the largest buyer in the world. The export of the Niger seed continuously increased. In Maharashtra, it is grown on an area of 0.141 lakh ha with the production of 0.023 lakh MT and productivity is $165 \mathrm{~kg} / \mathrm{ha}$ (2016-17). India tops in area, production and total export for Niger in the world.

Genetic diversity which is pre-requisite for any successful breeding programme is of paramount importance. Genetic divergence among the parents play a vital role in cultivar improvement because a cross involving genetically diverse parents is likely to generate more variability in segregating generations, and also which can be used for the desired improvement.

Generally, plant breeders select the parents on the basis of phenotypic diversity. Hence the knowledge of genetic diversity among the parents with respect to characters which are to be improved is essential. Therefore it is necessary to collect, conserve and study the genetic diversity among various crops in the form of germplasm for establishing the wide genetic base for the posterity. Keeping these things in the view, an effort has been made in the present study to evaluate a set of Niger genotypes with the objective to study the nature and magnitude of divergence among the genotypes of Niger.

\section{Materials and Methods}

The experimental material comprising forty five genotypes of Niger were grown in Randomized Block Design with two replications at the research farm of Department of Genetics and plant breeding, College of Agriculture, Dhule, during Kharif season of 2018. Each entry was represented by single row of $4.5 \mathrm{~m}$ length with spacing of 30 $\mathrm{cm}$ between rows. Data were recorded on five randomly and competitive plants of each genotype from each replication for twelve quantitative characters viz., days to $50 \%$ flowering, days to maturity, plant height $(\mathrm{cm})$, number of primary branches per plant, number of secondary branches per plant, number of capitula per plant, number of seeds per capitula, diameter of capitula $(\mathrm{cm}), 1000$ seed weight $(\mathrm{g})$, seed yield per plant $(\mathrm{g})$, protein content (g), oil content (g). Effective method suggested by Mahalanobis (1936) known as "Mahalanobis $\mathrm{D}^{2}$ statistics" or " $\mathrm{D}^{2}$ technique" is widely used to know genetic diversity in the germplasm. It was conducted to estimate the intra and inter cluster distances and to group the genotypes into different clusters and a logical grouping of genotypes following Tocher's method (Rao, 1952).

\section{Results and Discussion}

Analysis of variance for twelve characters indicated that the genotypes used in the present studies were found statistically significantly different (Table 1). The mean performances of 45 genotypes of Niger for twelve characters studied are presented in 
Table 2. The genotype DHLN-29 (61.50 days) was the earliest for flowering and days to maturity (91.50 days). The genotype DHLN$18(218.00 \mathrm{~cm})$ was found tall, genotypes DHLN-44 (30.00) exhibited significantly higher number of primary branches per plant, genotype DHLN-44 (76.30) produced maximum number of secondary branches per plant, genotype DHLN-44 (171.40) produced maximum number of capitula per plant, genotype DHLN-17 (40.7) recorded maximum number of seeds per capitula, genotype DHLN-41 (1.043 cm) recorded maximum diameter of capitula, genotype DHLN-5 (3.80 g) recorded maximum 1000 seed weight, genotype DHLN-42 (5.54 g) recorded highest seed yield, genotype DHLN-2 (37.10\%) recorded significantly high protein content, genotype DHLN-35 and DHLN-42 (42.22\%) recorded significantly high oil content.

On the basis of $\mathrm{D}^{2}$ values, the forty five genotypes evaluated for twelve characters were grouped into ten clusters by using the Tocher's method as described by Rao (1952). Cluster III was largest with 12 genotypes followed by cluster I (11 genotypes), cluster II and cluster V (6 genotypes), cluster VIII (5 genotypes), while clusters IV, VI, VII, IX and $\mathrm{X}$ were monogenotypic. In the present investigation grouping of genotypes into ten clusters suggested the presence of substantial amount of genetic diversity in the material under investigation. The clustering pattern and the eco-geographical regions of origin of each line are given in Table 1 and 3. Cluster III was the largest including 12 lines indicates that there was no association between clustering pattern and eco-geographical distribution of the cultures. Murty and Arunachalam (1966) and Somayajulu et al., (1970) while working with different crops, reported that geographical distribution does not necessary reflect genetic divergence. Cluster I which include 11 lines, cluster II and V include 6 lines and cluster VIII include 5 lines under study had varieties from different ecogeographical regions, thus supporting the view that geographic distribution and genetic divergence do not follow the same trend. Wide range of diversity was reported by many workers while evaluating niger genotypes Kumar (1999), Sreedhar et al., (2006), Patil (2007), Parmeshwarappa et al., (2011), Pulate et al., (2013), Khuntey and Kumar (2015), Pulate et al., (2015), Bisen et al., (2016) and Goyal and Bisen (2017).

The maximum intra cluster distance was observed for cluster $\mathrm{V}\left(\mathrm{D}^{2}=6.76\right)$ followed by cluster VIII $\left(\mathrm{D}^{2}=6.45\right)$ suggesting that genotypes present in these clusters might have different genetical architecture (Table 8). However, lowest intra cluster distance was observed in cluster $I\left(D^{2}=5.16\right)$ indicating that genotypes present in these cluster might have genetical similarities with one another and appeared to have evolved from common gene pool. Cluster IV, VI, VII, IX and X showed no intra cluster distance due to its monogenotypic nature.

Maximum inter cluster distance was observed between cluster II and X $\left(D^{2}=16.87\right)$ followed by cluster II and VIII $\left(\mathrm{D}^{2}=16.7\right)$, cluster II and IV $\left(D^{2}=15.77\right)$, cluster II and VII $\left(D^{2}=14.92\right)$, cluster $\mathrm{V}$ and $\mathrm{X}\left(\mathrm{D}^{2}=13.6\right)$, cluster II and III $\left(\mathrm{D}^{2}=12.65\right)$, cluster $\mathrm{V}$ and VII $\left(\mathrm{D}^{2}=12.39\right)$ cluster VI and X $\left(\mathrm{D}^{2}=12.13\right)$, cluster I and II $\left(\mathrm{D}^{2}=12.06\right)$, cluster II and IX $\left(\mathrm{D}^{2}=11.57\right)$, cluster II and V $\left(\mathrm{D}^{2}=11.52\right)$ cluster III and V $\left(\mathrm{D}^{2}=11.06\right)$, cluster VI and VIII $\left(\mathrm{D}^{2}=11.1\right)$, cluster $\mathrm{V}$ and VIII $\left(\mathrm{D}^{2}=10.95\right)$, cluster VIII and IX $\left(D^{2}=10.36\right)$, cluster IV and VI $\left(\mathrm{D}^{2}=10.18\right)$, cluster IV and V $\left(D^{2}=10.4\right)$, cluster VIII and $X\left(D^{2}=9.8\right)$, cluster VI and VII $\left(D^{2}=9.62\right)$, cluster $I$ and $X$ $\left(D^{2}=9.59\right)$, cluster $\mathrm{V}$ and $\mathrm{IX}\left(\mathrm{D}^{2}=9.38\right)$, cluster IV and IX $\left(\mathrm{D}^{2}=8.96\right)$, cluster III and VIII $\left(\mathrm{D}^{2}=8.93\right)$, cluster IX and $X\left(\mathrm{D}^{2}=8.83\right)$, cluster VII and IX $\left(D^{2}=8.82\right)$ cluster III and IV $\left(D^{2}=8.66\right)$, cluster III and $X\left(D^{2}=8.49\right)$, cluster 
VII and VIII $\left(\mathrm{D}^{2}=8.47\right)$, cluster $\mathrm{V}$ and VI $\left(\mathrm{D}^{2}=8.35\right)$, cluster I and $\mathrm{V}\left(\mathrm{D}^{2}=8.26\right)$, cluster I and VII $\left(\mathrm{D}^{2}=8.26\right)$ and cluster I and IV $\left(\mathrm{D}^{2}=8.22\right)$ indicating wide divergence among these clusters. These also suggest that genotype present in one cluster differ entirely from those presenting other clusters. The minimum inter cluster distance was found between cluster III and VI $\left(D^{2}=7.83\right)$, cluster III and IX $\left(D^{2}=7.64\right)$, cluster I and VIII $\left(\mathrm{D}^{2}=7.54\right)$, cluster IV and VIII $\left(\mathrm{D}^{2}=7.49\right)$, cluster II and VI $\left(D^{2}=7.45\right)$, cluster I and VI $\left(\mathrm{D}^{2}=7.42\right)$, cluster III and VII $\left(\mathrm{D}^{2}=7.1\right)$, cluster I and IX $\left(D^{2}=6.94\right)$, cluster I and III $\left(D^{2}=6.76\right)$, cluster IV and $X\left(D^{2}=6.62\right)$, cluster IV and VII $\left(D^{2}=5.37\right)$ and cluster VII and $X$ $\left(\mathrm{D}^{2}=4.64\right)$. The less inter cluster distance between these clusters revealed that genetic constitution of genotypes had close proximity.

Based on mean performance of clusters for 12 characters (Table 5). It was observed that cluster IX exhibited the highest protein content and was characterized by days to 50 per cent flowering, plant height, number of primary branches per plant, diameter of capitula, 1000 seed weight, days to maturity, number of secondary branches per plant, number of capitula per plant, seed yield per plant, oil content and number of seeds per capitula..

All these characters appeared to have played important role in determining seed yield of these cluster. Cluster II, IV and cluster IX showed nearly comparable seed yield. Cluster IV was characterized by less days to 50 per cent flowering, days to maturity, plant height, number of primary branches per plant, number of secondary branches per plant, diameter of capitula and number of capitula per plant.

Table.1 Analysis of variance for twelve characters in Niger

\begin{tabular}{|l|l|l|l|l|}
\hline $\begin{array}{l}\text { Sr. } \\
\text { No }\end{array}$ & Characters & \multicolumn{2}{l|}{ Mean sum of square } \\
\cline { 5 - 5 } & Replication & Genotype & Error \\
\hline $\mathbf{1}$ & Days to 50 per cent flowering & 0.100 & $95.622^{* *}$ & 7.622 \\
\hline $\mathbf{2}$ & Days to maturity & 5.877 & $65.018^{* *}$ & 26.673 \\
\hline $\mathbf{3}$ & Plant height (cm) & 740.173 & $11275.376^{* *}$ & 8486.941 \\
\hline $\mathbf{4}$ & No. of primary branches / plant & 2.116 & $20.370^{* *}$ & 5.074 \\
\hline $\mathbf{5}$ & No. of secondary branches / plant & 10.410 & $260.910^{* *}$ & 12.331 \\
\hline $\mathbf{6}$ & No. of capitula / plant & 105.408 & $1582.885^{* *}$ & 94.286 \\
\hline $\mathbf{7}$ & No. of seeds / plant & 1.534 & $49.349^{* *}$ & 8.527 \\
\hline $\mathbf{8}$ & Diameter of capitula (cm) & 0.009 & $0.011^{* *}$ & 0.005 \\
\hline $\mathbf{9}$ & 1000 seed weight $(\mathrm{g})$ & 0.047 & $0.462^{* *}$ & 0.072 \\
\hline $\mathbf{1 0}$ & Seed yield/ plant (g) & 0.065 & $1.108^{* *}$ & 0.258 \\
\hline $\mathbf{1 1}$ & Protein content $(\%)$ & 0.531 & $2.131^{* *}$ & 0.707 \\
\hline $\mathbf{1 2}$ & Oil content (\%) & 4.513 & $4.954^{* *}$ & 2.021 \\
\hline
\end{tabular}

*, ** Indicates significance at 5\% and 1\% level, respectively. 
Table.2 Mean performance of Niger genotype

\begin{tabular}{|c|c|c|c|c|c|c|c|c|c|c|c|c|c|}
\hline Sr. No & Genotypes & $\begin{array}{c}\text { Days to } 50 \\
\text { per cent } \\
\text { flowering }\end{array}$ & $\begin{array}{c}\text { Days to } \\
\text { maturity }\end{array}$ & $\begin{array}{c}\text { Plant } \\
\text { height } \\
(\mathrm{cm})\end{array}$ & $\begin{array}{c}\text { No. of } \\
\text { primary } \\
\text { branches } \\
\text { / plant }\end{array}$ & $\begin{array}{c}\text { No of } \\
\text { secondary } \\
\text { branches } \\
\text { plant }\end{array}$ & $\begin{array}{c}\text { No. of } \\
\text { capitula } \\
\text { / plant }\end{array}$ & $\begin{array}{c}\text { No of } \\
\text { seeds / } \\
\text { capitula }\end{array}$ & $\begin{array}{c}\text { Diameter } \\
\text { of capitula } \\
\text { (cm) }\end{array}$ & $\begin{array}{c}1000 \\
\text { seed } \\
\text { weight } \\
(\mathrm{g})\end{array}$ & $\begin{array}{r}\text { Seed } \\
\text { yield /plant } \\
\text { (g) }\end{array}$ & $\begin{array}{c}\text { Protein } \\
\text { content }(\%)\end{array}$ & $\begin{array}{c}\text { Oil } \\
\text { content } \\
(\%)\end{array}$ \\
\hline 1 & DHLN-1 & 75.5 & 108.0 & 190.6 & 26.1 & 50.9 & 108.6 & 31.1 & 0.855 & 2.31 & 4.63 & 34.83 & 41.07 \\
\hline 2 & DHLN-2 & 71.0 & 104.5 & 193.9 & 22.2 & 34.3 & 71.4 & 31.8 & 0.856 & 2.48 & 3.58 & 37.10 & 39.01 \\
\hline 3 & DHLN-3 & 66.5 & 99.5 & 195.2 & 25.1 & 48.5 & 129.1 & 34.7 & 0.985 & 2.59 & 5.29 & 34.40 & 41.40 \\
\hline 4 & DHLN-4 & 76.5 & 105.0 & 190.7 & 21.5 & 43.1 & 84.8 & 32.1 & 0.882 & 2.26 & 4.18 & 36.81 & 38.05 \\
\hline 5 & DHLN-5 & 83.0 & 112.5 & 207.9 & 28.0 & 46.0 & 135.8 & 29.7 & 0.997 & 3.80 & 4.35 & 35.12 & 40.58 \\
\hline 6 & DHLN-6 & 74.5 & 102.5 & 212.6 & 24.4 & 37.3 & 102.1 & 24.1 & 0.720 & 2.15 & 3.49 & 34.85 & 41.25 \\
\hline 7 & DHLN-7 & 78.5 & 107.0 & 195.3 & 17.6 & 30.8 & 57.7 & 23.2 & 0.873 & 1.79 & 3.59 & 36.00 & 39.22 \\
\hline 8 & DHLN-8 & 84.5 & 106.5 & 196.8 & 23.4 & 44.7 & 110.2 & 26.9 & 0.856 & 2.09 & 3.35 & 35.20 & 40.72 \\
\hline 9 & DHLN-9 & 85.0 & 112.5 & 191.3 & 20.0 & 35.9 & 80.7 & 23.7 & 0.958 & 2.51 & 2.85 & 34.38 & 38.43 \\
\hline 10 & DHLN10 & 77.0 & 105.5 & 167.5 & 21.9 & 28.3 & 48.1 & 26.3 & 0.980 & 1.59 & 3.32 & 35.76 & 40.04 \\
\hline 11 & DHLN-11 & 73.5 & 102.5 & 172.5 & 18.2 & 29.7 & 86.1 & 28.4 & 0.792 & 2.33 & 4.27 & 35.46 & 42.04 \\
\hline 12 & DHLN-12 & 79.5 & 109.5 & 206.5 & 26.1 & 58.0 & 149.3 & 26.0 & 0.968 & 2.45 & 4.83 & 35.02 & 37.58 \\
\hline 13 & DHLN-13 & 77.0 & 109.0 & 175.7 & 24.9 & 45.0 & 79.6 & 30.3 & 0.946 & 3.05 & 4.10 & 35.10 & 37.87 \\
\hline 14 & DHLN-14 & 78.5 & 107.5 & 189.2 & 21.2 & 30.3 & 105.3 & 30.3 & 0.815 & 2.09 & 3.04 & 33.91 & 39.19 \\
\hline 15 & DHLN-15 & 73.0 & 102.5 & 201.8 & 25.7 & 47.4 & 109.3 & 29.4 & 0.810 & 1.95 & 3.19 & 34.59 & 40.68 \\
\hline 16 & DHLN-16 & 88.0 & 116.5 & 186.9 & 26.8 & 40.7 & 91.5 & 21.0 & 0.889 & 1.96 & 3.00 & 33.32 & 37.48 \\
\hline 17 & DHLN-17 & 82.0 & 113.0 & 192.2 & 21.4 & 40.3 & 101.6 & 40.7 & 0.937 & 2.84 & 3.96 & 34.85 & 40.53 \\
\hline 18 & DHLN-18 & 83.0 & 108.0 & 214.3 & 26.9 & 39.2 & 85.9 & 19.9 & 0.796 & 2.21 & 4.09 & 35.07 & 40.20 \\
\hline 19 & DHLN-19 & 73.5 & 102.0 & 194.4 & 21.0 & 33.1 & 95.3 & 24.0 & 0.873 & 2.07 & 3.24 & 35.26 & 37.74 \\
\hline 20 & DHLN-20 & 81.0 & 108.5 & 196.3 & 22.9 & 40.0 & 93.5 & 37.5 & 0.823 & 1.88 & 3.27 & 35.47 & 39.54 \\
\hline 21 & DHLN-21 & 71.5 & 100.0 & 169.4 & 20.1 & 32.4 & 110.3 & 31.8 & 0.853 & 2.72 & 4.64 & 34.93 & 41.69 \\
\hline 22 & DHLN-22 & 67.5 & 100.0 & 179.6 & 23.3 & 43.3 & 87.9 & 31.3 & 0.993 & 1.95 & 3.56 & 33.58 & 39.54 \\
\hline 23 & DHLN-23 & 78.5 & 105.0 & 189.6 & 23.4 & 43.1 & 83.5 & 22.7 & 0.918 & 2.04 & 3.53 & 34.77 & 39.01 \\
\hline 24 & DHLN-24 & 63.0 & 99.5 & 179.0 & 18.8 & 38.0 & 108.6 & 30.3 & 0.911 & 1.82 & 2.98 & 33.35 & 38.53 \\
\hline 25 & DHLN-25 & 70.5 & 98.5 & 190.1 & 23.9 & 30.4 & 82.0 & 21.1 & 0.940 & 2.03 & 3.89 & 34.75 & 39.38 \\
\hline 26 & DHLN-26 & 56.0 & 98.0 & 161.9 & 21.9 & 37.3 & 91.7 & 24.7 & 0.908 & 1.63 & 2.93 & 34.34 & 40.36 \\
\hline
\end{tabular}




\begin{tabular}{|c|c|c|c|c|c|c|c|c|c|c|c|c|c|}
\hline 27 & DHLN-27 & 76.0 & 105.0 & 186.3 & 23.6 & 40.1 & 97.6 & 23.3 & 1.002 & 2.75 & 3.75 & 33.17 & 38.51 \\
\hline 28 & DHLN-28 & 65.0 & 101.0 & 196.1 & 25.9 & 53.1 & 132.5 & 29.0 & 0.979 & 2.75 & 4.03 & 34.01 & 39.14 \\
\hline 29 & DHLN-29 & 61.5 & 91.5 & 196.7 & 22.3 & 30.8 & 81.2 & 32.7 & 0.920 & 1.77 & 3.64 & 33.10 & 38.78 \\
\hline 30 & DHLN-30 & 82.5 & 111.0 & 194.0 & 27.2 & 39.5 & 106.8 & 27.3 & 0.965 & 1.94 & 2.86 & 34.32 & 38.85 \\
\hline 31 & DHLN-31 & 73.0 & 99.5 & 197.3 & 20.2 & 32.4 & 108.2 & 29.7 & 0.794 & 2.45 & 4.27 & 33.53 & 38.12 \\
\hline 32 & DHLN-32 & 71.5 & 97.0 & 185.8 & 23.8 & 40.9 & 90.4 & 32.3 & 0.906 & 1.74 & 3.12 & 33.76 & 41.39 \\
\hline 33 & DHLN-33 & 80.5 & 109.0 & 188.4 & 18.6 & 31.7 & 89.9 & 36.4 & 0.884 & 3.03 & 2.74 & 32.58 & 41.96 \\
\hline 34 & DHLN-34 & 72.5 & 103.5 & 188.6 & 23.4 & 33.8 & 94.4 & 19.9 & 0.851 & 2.18 & 4.53 & 33.12 & 38.38 \\
\hline 35 & DHLN-35 & 67.5 & 103.5 & 182.2 & 17.8 & 37.7 & 108.4 & 27.2 & 1.011 & 2.14 & 3.96 & 33.11 & 42.22 \\
\hline 36 & DHLN-36 & 68.0 & 96.0 & 189.4 & 19.4 & 34.7 & 64.4 & 16.7 & 0.900 & 1.66 & 2.62 & 33.28 & 38.74 \\
\hline 37 & DHLN-37 & 79.0 & 108.0 & 199.1 & 29.1 & 59.7 & 152.0 & 27.4 & 0.978 & 2.21 & 3.70 & 33.34 & 37.07 \\
\hline 38 & DHLN-38 & 74.5 & 104.5 & 180.6 & 21.2 & 45.4 & 122.1 & 29.2 & 1.001 & 1.91 & 4.10 & 33.53 & 36.34 \\
\hline 39 & DHLN-39 & 72.0 & 102.0 & 185.6 & 23.1 & 38.2 & 99.9 & 27.2 & 0.968 & 3.09 & 3.94 & 35.34 & 40.11 \\
\hline 40 & DHLN-40 & 78.0 & 105.5 & 166.5 & 21.6 & 39.8 & 119.0 & 27.8 & 0.982 & 2.45 & 3.87 & 33.49 & 37.24 \\
\hline 41 & DHLN-41 & 78.0 & 107.0 & 194.4 & 24.3 & 60.0 & 148.3 & 30.6 & 1.043 & 3.06 & 5.51 & 34.59 & 37.12 \\
\hline 42 & DHLN-42 & 73.5 & 106.0 & 199.3 & 26.5 & 53.4 & 159.0 & 26.0 & 1.030 & 3.04 & 5.54 & 34.38 & 42.22 \\
\hline 43 & DHLN-43 & 80.0 & 113.0 & 185.2 & 29.5 & 75.6 & 159.4 & 32.4 & 0.999 & 2.71 & 5.19 & 33.53 & 39.74 \\
\hline 44 & DHLN-44 & 84.5 & 119.5 & 193.1 & 30.0 & 76.3 & 171.4 & 39.3 & 0.988 & 2.68 & 4.54 & 32.87 & 37.05 \\
\hline \multirow[t]{5}{*}{45} & DHLN-45 & 86.0 & 114.5 & 195.0 & 27.1 & 64.9 & 148.4 & 27.9 & 0.976 & 2.47 & 3.29 & 33.68 & 38.57 \\
\hline & G. Mean & 75.36 & 105.34 & 189.88 & 23.36 & 42.58 & 105.40 & 28.48 & 0.91 & 2.32 & 3.83 & 34.38 & 39.39 \\
\hline & S.E. \pm & 1.95 & 3.65 & 9.82 & 1.59 & 2.48 & 6.86 & 2.06 & 0.05 & 0.19 & 0.35 & 0.59 & 1.00 \\
\hline & C.D. at $5 \%$ & 5.56 & 10.40 & 27.96 & 4.53 & 7.07 & 19.56 & 5.88 & 0.15 & 0.54 & 1.02 & 1.69 & 2.86 \\
\hline & C.V. (\%) & 3.66 & 4.90 & 7.31 & 9.64 & 8.24 & 9.21 & 10.25 & 8.27 & 11.60 & 13.26 & 2.44 & 3.60 \\
\hline
\end{tabular}


Table. 3 Grouping of forty five Niger genotypes into different clusters

\begin{tabular}{|c|c|c|c|}
\hline $\begin{array}{l}\text { Sr. } \\
\text { No. }\end{array}$ & Cluster & $\begin{array}{ll}\text { No. } & \text { of } \\
\text { genotypes }\end{array}$ & Name of genotypes \\
\hline 1 & I & 11 & $\begin{array}{l}\text { DHLN-1, DHLN-15, DHLN-32, DHLN-22, DHLN-6, } \\
\text { DHLN-36, DHLN-27, DHLN-23, DHLN-19, DHLN- } \\
\text { 39, DHLN-13 }\end{array}$ \\
\hline 2 & II & 6 & $\begin{array}{l}\text { DHLN-12, DHLN-37, DHLN-41, DHLN-45, DHLN- } \\
\text { 43, DHLN-44 }\end{array}$ \\
\hline 3 & III & 12 & $\begin{array}{l}\text { DHLN-8, DHLN-20, DHLN-16, DHLN-9, DHLN-30, } \\
\text { DHLN-18, DHLN-7, DHLN-4, DHLN-14, DHLN-40, } \\
\text { DHLN-31, DHLN-11 }\end{array}$ \\
\hline 4 & IV & 1 & DHLN-21 \\
\hline 5 & $\mathrm{~V}$ & 6 & $\begin{array}{l}\text { DHLN-24, DHLN-35, DHLN-3, DHLN-26, DHLN-28, } \\
\text { DHLN-42 }\end{array}$ \\
\hline 6 & VI & 1 & DHLN-38 \\
\hline 7 & VII & 1 & DHLN-17 \\
\hline 8 & VIII & 5 & DHLN-25, DHLN-29, DHLN-34, DHLN-2, DHLN-10 \\
\hline 9 & IX & 1 & DHLN-5 \\
\hline 10 & $\mathrm{X}$ & 1 & DHLN-33 \\
\hline
\end{tabular}

Table. 4 Average intra and inter cluster distance ( $D^{2}$ values) for twelve characters in Niger

\begin{tabular}{|l|l|l|l|l|l|l|l|l|l|l|}
\hline Clusters & I & II & III & IV & V & VI & VII & VIII & IX & X \\
\hline I & $\mathbf{5 . 1 6}$ & 12.06 & 6.76 & 8.22 & 8.26 & 7.42 & 8.26 & 7.54 & 6.94 & 9.59 \\
\hline II & & $\mathbf{5 . 4 9}$ & 12.65 & 15.77 & 11.52 & 7.45 & 14.92 & 16.7 & 11.57 & 16.87 \\
\hline III & & & $\mathbf{6 . 0 5}$ & 8.71 & 11.06 & 7.83 & 7.1 & 8.93 & 7.64 & 8.49 \\
\hline IV & & & & $\mathbf{0}$ & 10.4 & 10.18 & 5.37 & 7.49 & 8.96 & 6.62 \\
\hline $\mathbf{V}$ & & & & & 6.76 & 8.35 & 12.39 & 10.95 & 9.38 & 13.6 \\
\hline VI & & & & & & $\mathbf{0}$ & 9.62 & 11.1 & 8.66 & 12.13 \\
\hline VII & & & & & & $\mathbf{0}$ & 8.47 & 8.82 & 4.64 \\
\hline VIII & & & & & & & & $\mathbf{6 . 4 5}$ & 10.36 & 9.8 \\
\hline IX & & & & & & & & & $\mathbf{0}$ & 8.83 \\
\hline $\mathbf{X}$ & & & & & & & & & & $\mathbf{0}$ \\
\hline
\end{tabular}


Table.5 Cluster means for twelve characters in ten clusters of forty five Niger genotypes

\begin{tabular}{|c|c|c|c|c|c|c|c|c|c|c|c|c|}
\hline Sr. & Characters & & & & & & Cluster & average & & & & \\
\hline No & & I & II & III & IV & $\mathbf{V}$ & VI & VII & VIII & IX & $\mathbf{X}$ & Average \\
\hline 1 & Days to 50 per cent flowering & 73.36 & 81.17 & 80.17 & 71.50 & 65.25 & 74.50 & 82.00 & 70.50 & 83.00 & 80.50 & 76.19 \\
\hline 2 & Days to maturity & 102.64 & 111.92 & 107.50 & 100.00 & 101.25 & 104.50 & 113.00 & 100.70 & 112.50 & 109.00 & 106.30 \\
\hline 3 & Plant height $(\mathrm{cm})$ & 190.13 & 195.54 & 190.93 & 169.45 & 185.63 & 180.60 & 192.20 & 187.36 & 207.90 & 188.40 & 188.81 \\
\hline 4 & No. of primary branches/plant & 23.52 & 27.68 & 22.29 & 20.10 & 22.67 & 21.20 & 21.40 & 22.74 & 28.00 & 18.60 & 22.82 \\
\hline 5 & No. of secondary branches/plant & 41.28 & 65.77 & 37.18 & 32.45 & 44.67 & 45.40 & 40.30 & 31.52 & 46.00 & 31.70 & 41.62 \\
\hline 6 & No. of capitula/plant & 92.60 & 154.81 & 94.14 & 110.30 & 121.55 & 122.10 & 101.60 & 75.41 & 135.80 & 89.90 & 109.82 \\
\hline 7 & No. of seeds / capitula & 26.93 & 30.60 & 26.96 & 37.50 & 25.85 & 29.20 & 40.70 & 30.76 & 29.70 & 32.30 & 31.05 \\
\hline 8 & Diameter of capitula $(\mathrm{cm})$ & 0.90 & 0.99 & 0.87 & 0.85 & 0.97 & 1.00 & 0.94 & 0.91 & 1.00 & 0.88 & 0.93 \\
\hline 9 & 1000 seed weight $(\mathrm{g})$ & 2.25 & 2.60 & 2.16 & 2.72 & 2.33 & 1.91 & 2.85 & 2.01 & 3.81 & 3.03 & 2.56 \\
\hline 10 & seed yield/plant (g) & 3.56 & 4.51 & 3.55 & 4.64 & 4.12 & 4.10 & 3.97 & 3.79 & 4.35 & 2.74 & 3.93 \\
\hline 11 & Protein content $(\%)$ & 34.41 & 33.84 & 34.75 & 34.93 & 33.93 & 33.53 & 34.85 & 34.78 & 35.12 & 32.58 & 34.27 \\
\hline 12 & Oil content $(\%)$ & 39.63 & 37.86 & 39.10 & 41.69 & 40.65 & 36.34 & 40.53 & 39.12 & 40.58 & 41.96 & 39.74 \\
\hline
\end{tabular}


Table.6 Relative percent contribution of different characters towards total genetic divergence in Niger.

\begin{tabular}{|l|l|l|l|}
\hline Sr. No. & Characters & $\begin{array}{l}\text { No. of times } \\
\text { ranked 1 }\end{array}$ & $\begin{array}{l}\text { Percent } \\
\text { contribution }\end{array}$ \\
\hline $\mathbf{1}$ & Days to 50 per cent flowering & 54 & $5.45 \%$ \\
\hline $\mathbf{2}$ & Days to maturity & 1 & $0.10 \%$ \\
\hline $\mathbf{3}$ & Plant height (cm) & 0 & $0.00 \%$ \\
\hline $\mathbf{4}$ & No. of primary branches/plant & 134 & $13.54 \%$ \\
\hline $\mathbf{5}$ & No. of secondary branches /plant & 161 & $16.26 \%$ \\
\hline $\mathbf{6}$ & No. of capitula/plant & 122 & $12.32 \%$ \\
\hline $\mathbf{7}$ & No. of seeds / capitula & 294 & $29.70 \%$ \\
\hline $\mathbf{8}$ & Diameter of capitula (cm) & 6 & $0.61 \%$ \\
\hline $\mathbf{9}$ & 1000 seed weight (g) & 124 & $12.53 \%$ \\
\hline $\mathbf{1 0}$ & Seed yield/plant $(\mathrm{g})$ & 31 & $3.13 \%$ \\
\hline $\mathbf{1 1}$ & Protein content $(\%)$ & 23 & $2.32 \%$ \\
\hline $\mathbf{1 2}$ & Oil content $(\%)$ & 40 & $4.04 \%$ \\
\hline
\end{tabular}

Fig. 1 Clustering by Tocher method

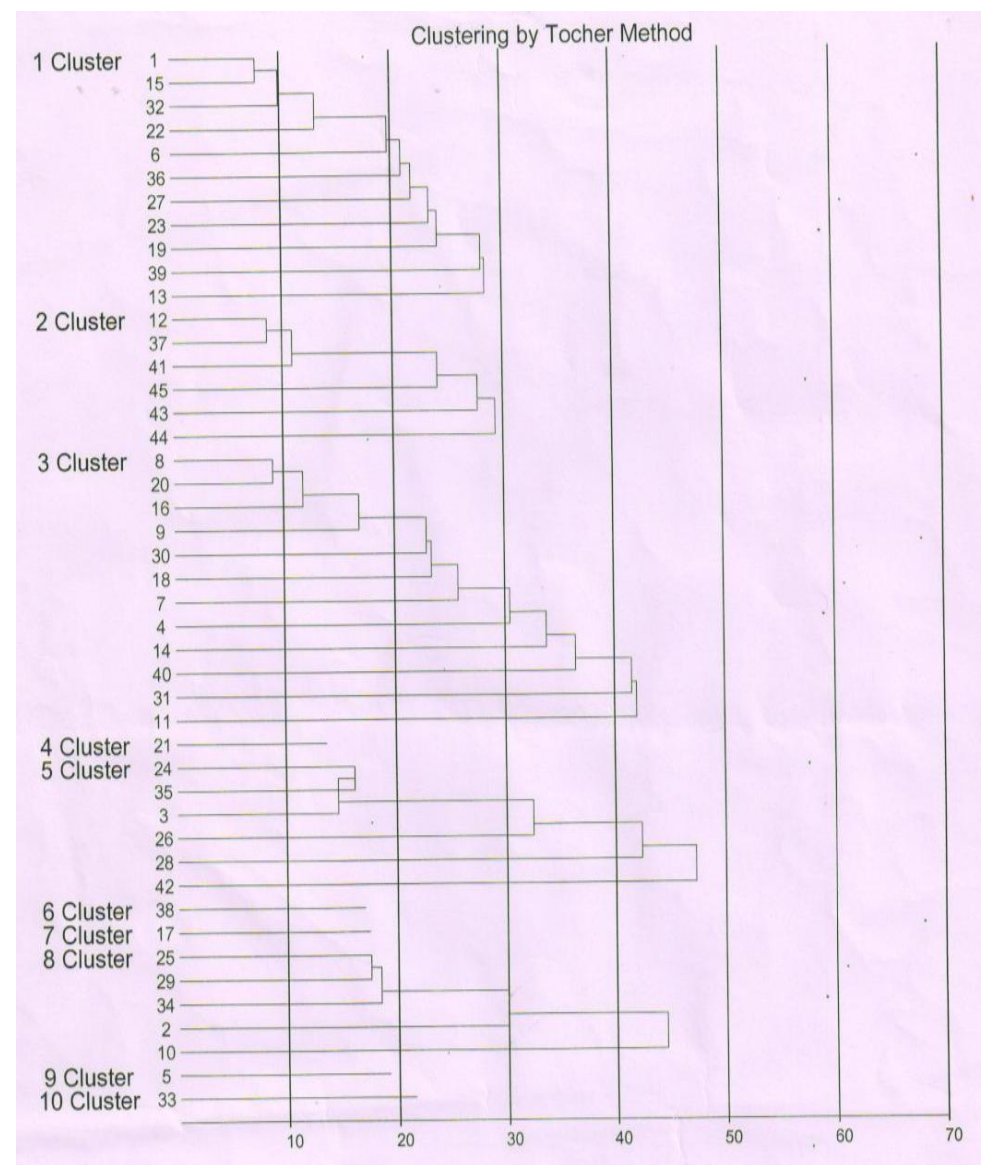


Fig .2 Cluster diagram of forty five germplasm in Niger.

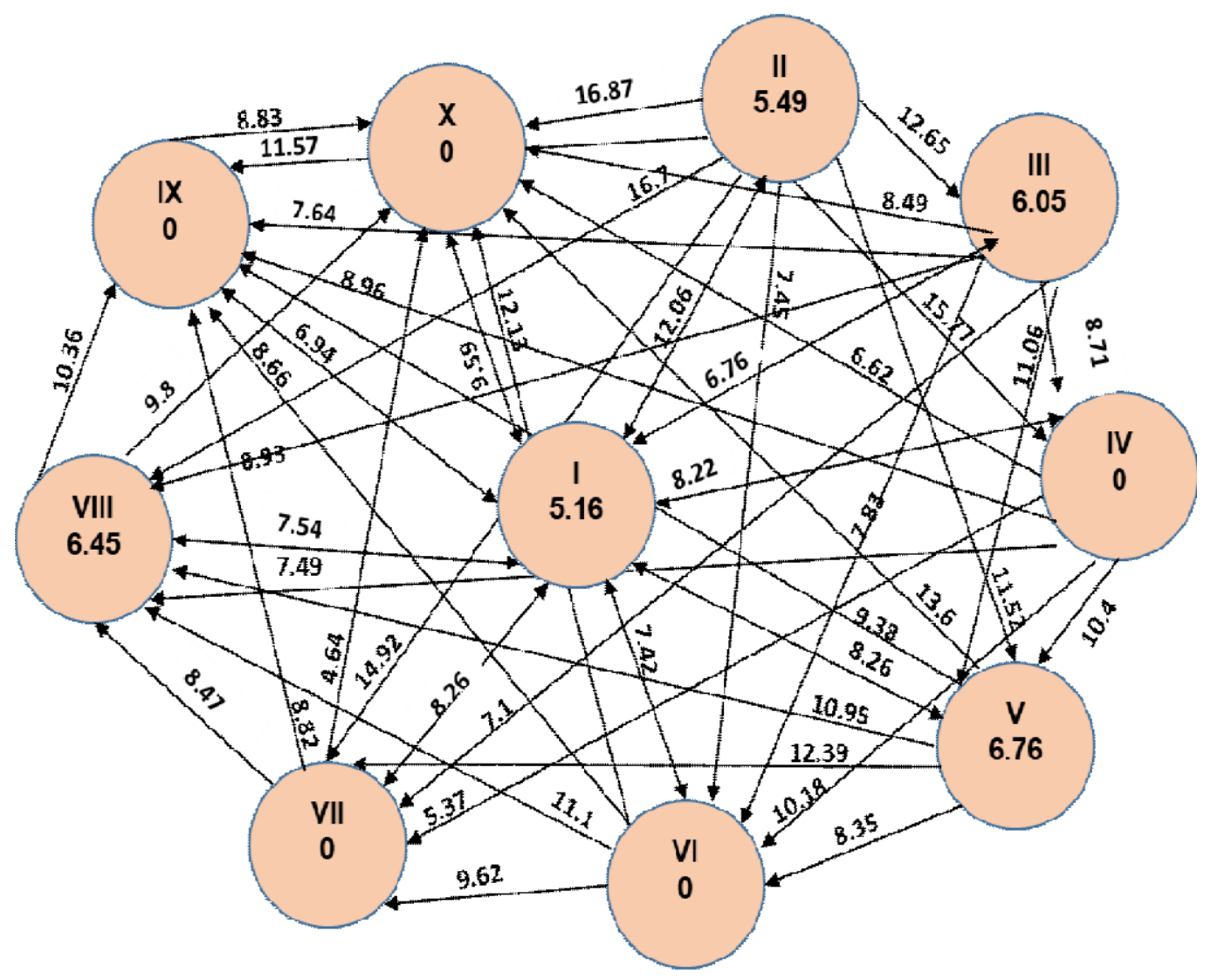

Fig.3 Divergence classes

\begin{tabular}{lllllr} 
DC 4 & \multicolumn{1}{c}{ DC 3 } & \multicolumn{2}{c}{ DC 2 } & \multicolumn{2}{c}{ DC 1 } \\
$\qquad$ & $\downarrow$ & $\downarrow$ & $\downarrow$ & & $\downarrow$ \\
$\mathrm{X}$ & $\mathrm{m}-\mathrm{s}$ & $\mathrm{M}$ & $\mathrm{m}+\mathrm{s}$ & $\mathrm{Y}$ \\
$(4.64)$ & $(6.50)$ & $(9.37)$ & $(12.24)$ & & $(16.87)$
\end{tabular}

$\mathrm{M}=$ The mean of ten clusters and five intra-clusters (as monogenotypic cluster IV, VI, VII, IX and X had no intracluster distance) was 9.37 .

$\mathrm{X}=$ Minimum value of distance 4.64 .

$\mathrm{Y}=$ Maximum value of distance 16.87 .

$\mathrm{S}=$ Standard deviation 2.87 .

$\mathrm{m}-\mathrm{s}=$ Mean - Standard deviation.

$\mathrm{m}+\mathrm{s}=$ Mean + Standard deviation.

Similarly cluster $\mathrm{X}$ was characterized by less number of primary branches per plant, number of capitula per plant, seed yield per plant, protein content, diameter of capitula, number of secondary branches per plant and plant height. Similarly cluster I, cluster IV, cluster VI and cluster VIII show less cluster mean for most of the characters.

Cluster V was characterized by high diameter 
of capitula, oil content, seed yield per plant, number of secondary branches per plant, number of capitula per plant and number of primary branches per plant. On the basis of mean performance of different clusters, it was observed that cluster X, II, V, VII and cluster IV were performing well for most of the characteristics.

The variance of cluster mean provides information on relative importance of different characters towards grain yield. The present study revealed that number of seeds per capitula $(29.70 \%)$ contributed more to genetic diversity as reflected from the Table 6 . Which was followed by number of secondary branches per plant (16.26\%), number of primary branches per plant (13.54\%), 1000 seed weight $(12.53 \%)$, number of capitula per plant $(12.32 \%)$, days to 50 per cent flowering $(5.45 \%)$, oil content $(4.04 \%)$, seed yield per plant $(3.13 \%)$, protein content $(2.32 \%)$, diameter of capitula $(0.61 \%)$, days to maturity $(0.10 \%)$ and last was plant height $(0.00 \%)$.

Among the 12 characters studied the most important character contributing to the divergence was number of seeds per capitula followed by number of secondary branches per plant, number of primary branches per plant, 1000 seed weight, number of capitula per plant. It is almost close to result reported by Patil (2007), Pulate et al., (2015).

The magnitude of contribution by plant height, days to maturity, diameter of capitula, protein content, seed yield per plant, oil content, days to 50 per cent flowering was low.

The mean of ten clusters and five intraclusters (as monogenotypic cluster IV, VI, VII, IX and X had no intra cluster distance) was 9.37 and standard deviation 2.87. The minimum $(\mathrm{X})$ and maximum $(\mathrm{Y})$ values among these distances were 4.64 and 16.87 respectively. Grouping of cluster pairs into the divergence class (DC) are presented in Table 6. On the light of discussion, initial choice of parents should be made from the cluster combinations falling in the divergence classes DC2 and DC3.

While crossing among the genotypes of a cluster, the per se performance of the genotypes for different trait such as earliness (days to 50 per cent flowering and days to maturity), plant height, number of capitula per plant, number of seeds per capitula, 1000 seed weight, seed yield per plant etc. should be taken into account. So, those desirable transgressive segregants would be obtained after hybridization. The present study revealed no parallelism between genetic divergence and geographical distribution of genotypes which was demonstrated by grouping of genotypes from same origin into different clusters separated by high genetic distance. This suggested, that genetic drift and selection in different environments may cause geographical distances. Considering intercluster distances, cluster mean and per se performance, and divergence class the genotypes viz., DHLN-17, DHLN-18, DHLN26, DHLN-29, DHLN-39, DHLN-41, DHLN42, DHLN-44 were distinct and diverse and can be classified as promising genotypes. These genotypes can be used for intercrossing to obtain heterosis and also wider variability in Niger.

Hybridization between the genotypes of cluster II with the genotypes of cluster X may result in exploiting more heterosis with maximum genetic divergence and are likely to produce desirable transgressive segregants in segregating generations for further crop improvement.

\section{References}

Arunachalam,V. and A. Bandopadhay. 1984. Limits to genetic divergence for 
occurrence of heterosis experimental evidence from crop plants. Indian Journal of Genetics,44 (3): 548-554.

Bain, A. S. and K. C. Sood. 1984. Resolution of genetic divergence for choice of parents in soybean breeding. Crop Improvement, 11(1): 20-24.

Bisen R., A. K. Panday, S. Jain, R. Sahu and M. Malviya (2016) Estimation of genetic divergence among the Niger germplasm. The Journal of Animal and Plant Sciences, 26 (5):1320-1325. ISSN: 1018-7081.

Goyal V. K. and RajaniBisen. 2017. Assessment of genetic divergence in niger germplasm. International Journal of Chemical Studies, 5(4): 1482-1485.

Khuntey Y. and N. Kumar. 2015. Systematic analysis of genotypic diversity in niger (Guizotia abyssinica(L.) Cass). Indian Research Journal of Genetics \& Biotechnology 7 (3): 355-358.

Kumar, S. 1999. Genetic divergence and Correlation studies in Niger (Guizotia abyssinica (L.f.) Cass) M. Sc. (Agri.) Thesis, submitted to Birsa Agricultural University, Kanke (Ranchi-6), Ex. Bihar state now Chhattisgarh state, India.

Mahalanobis, P. C. 1936. On the Genaralised Distance In Science, India. 2: 49-55.

Murty, B. R. and V. Arunachalam. 1966. The nature of divergence in relation to breeding system in some crop plants. Indian Journal of Genetics and Plant Breeding, 26: 188-198.
Parameshwarappa, S. G., M. G. Palakshappa and Gayatree G.Shinde.2011. Genetic diversity studies in germplasm collections of Niger (Guizotia abyssinica (L.f.) Cass). Plant Archives Vol., 11 (2): pp. 1071-1074.

Patil, H. S. 2007. Multivariate analysis as aid to genotype selection for breeding in Niger (Guizotia abyssinica (L.f.) Cass). Indian Journal Crop Improvement Vol., 34 (2): 202- 205.

Pulate,S. C., H. S. Patil and M. R. Patil. (2013). Multivariate analysis of genetic divergence among niger genotypes in relation to seed oil quality traits. The Bioscan- International Journal of Life Sciences, 8 (3): 829833.

Pulate, S. C., H. S. Patil, N. M. Mangar and M. R. Patil. 2015. Genetic variability and diversity in Niger (Guizotiaabyssinica (L.f.)cass). Bioinfolet- Journal of Life Science, 12 (1):280-284.

Rao, C. R. 1952. Advanced statistical methods in Biometrical Research. John Willey and Sons. Inc., New York.

Somayajulu, P. L., A.B. Joshi and B. R. Murty. 1970. Genetic divergence in wheat. Indian Journal of Genetics and Plant Breeding, 30: 47-48.

Sreedhar, R. V., S. Gangaprasad S, R. L. Ravikumar and P. M. Salimath. 2006. Assessment of genetic diversity in niger, (Guizotia abyssinica (L.f.) Cass) Journal of Oilseed Research, B.A.U., 23 (2): 191-193.

\section{How to cite this article:}

Shubhangi G.Patil, V. V. Bhavsar, Shweta D. Deokar and V. S. Girase 2019. Genetic Divergence in Niger (Guizotia abyssinica (L.f) Cass). Int.J.Curr.Microbiol.App.Sci. 8(09): 1891-1902. doi: https://doi.org/10.20546/ijcmas.2019.809.219 